$0,7 \mathrm{~cm}$. Posee un aspecto isointenso en T1 y ligeramente hiperintenso en T2 con zonas de ligera hipointensidad en su interior (Fig. 1). Dicha lesión provoca una compresión medular con reducción del diámetro anteroposterior del canal raquídeo. El análisis de sangre realizado no mostró alteraciones en la coagulación. Se interviene con carácter de urgencia procediendo a la resección de un gran hematoma epidural en la localización mencionada, no hallando malformaciones vasculares ni proceso neoplásico subyacente (1-5). El paciente pudo ser extubado a las 12 horas de su intervención y dado de alta a planta en 48 horas sin déficits neurológicos.

El hematoma epidural espontáneo sin existencia de traumatismo previo ni de coagulopatía adquirida constituye una entidad excepcional $(1,4,6,7)$, que provoca isquemia medular aguda por compresión de la misma por parte de dicho hematoma, cuyo diagnóstico se realiza mediante RNM $(2,7,8)$, siendo la descompresión precoz el único tratamiento que ha mostrado ser eficaz $(2,4,8)$.

B. Obón Azuara, I. Gutiérrez Cía, B. Villanueva Anadón, P. Cía Blasco, R. Montoiro Allué, A. Cárcamo Merino

Servicio de Medicina Intensiva. Hospital Clínico Universitario. Zaragoza

1. Vázquez-Barquero A, Abascal F, García-Valtuille R, Pinto JI, Figols FJ, Cereza L. Chronic nontraumatic spinal epidural hematoma of the lumbar spine: MRI diagnosis. Eur Radiol 2000; 10: 1602-5.

2. Alexiadou -Rudolf C, Ernestus RI, Nanassis K, Lanfermann H, Klug N. Acute nontraumatic spinal epidural hematomas. An important differential diagnosis in spinal emergencies. Spine 1998; 23: 1810-3.

3. Akutsu H, Sugita K, Sonobe M, Matsumura A. A case of nontraumatic spinal epidural hematoma caused by extradural varix: consideration of etiology. Spine J 2003; 3: 534-8.

4. Lonjon MM, Paquis P, Chanalet S, Grellier P. Nontraumatic spinal epidural hematoma: report of four cases and review of the literature. Neurosurgery 1997; 41: 483-6.

5. Kirwan R, Saigal G, Faingold R, O'Gorman A. Nontraumatic acute and subacute enhancing spinal epidural hematoma mimicking a tumor in a child. Pediatr Radiol 2004; 34: 499-502.

6. Lin IY. Diagnostic pitfall: nontraumatic spinal epidural hematoma mimicking a brainstem stroke. Ann Emerg Med 2004; 44: 183-4.

7. Lovblad KO, Baumgartner RW, Zambaz BD, Remonda L, Ozdoba C, Schorth G. Nontraumatic spinal epidural hematomas. MR features. Acta Radiol 1997; 38: 8-13.

8. Awada A, Russell N, al Fayez N, Naufal R, Al Kohlani H. Spontaneous cervical epidural hematoma: case report. Spinal Cord 1998; 36: 71-2.

\section{Afasia de expresión como forma de presentación de una meningitis por Listeria monocytogenes}

\section{Sr. Director:}

Listeria monocytogenes es un bacilo gram positivo, aerobio y no esporulado, con un especial tropismo por las membranas meníngeas. Afecta, por lo general, a niños y ancianos, o a pacientes con inmunodepresión celular (1), aunque en los últimos años se ha descrito en pacientes de mediana edad (2) y en niños inmunocompetentes (3). Su presentación con afasia es extremadamente rara $(4,5)$. Se presenta el caso de una paciente de 71 años, VIH positiva y hepatópata crónica, que consultó por cefalea, rigidez de nuca y afasia de expresión y cuyo diagnóstico final fue de meningitis por Listeria monocytogenes.

Mujer de 71 años, con antecedentes de infección VIH categoría A3, hemorragia digestiva por varices esofágicas y descompensación ascítico-edematosa secundaria a granulomatosis hepática crónica. En el estudio de la hepatopatía se descartó neoplasia y causas infecciosas, víricas e inmunes. La exploración mostró fiebre de $38,6^{\circ} \mathrm{C}$, Glasgow 13 , rigidez de nuca, afasia de expresión y ascitis. Analítica: glucosa $96 \mathrm{mg} / \mathrm{dl}$, hemoglobina 9,2 mg/dl, VCM 82,4 fl, tiempo de protrombina 59,2\%, resto dentro de la normalidad. TC craneal: sin alteraciones destacables. Punción lumbar (PL): 43 células nucleadas $/ \mathrm{mm}^{3}$ (78\% neutrófilos), glucosa $33 \mathrm{mg} / \mathrm{dl}$, proteínas $109 \mathrm{mg} / \mathrm{dl}$ y ADA 20,8 UI/l, tinta china negativa. Paracentesis: líquido con características de trasudado con cultivos negativos. Previo a la PL se inició tratamiento endovenoso con Ceftriaxona 2 gramos cada 12 horas y Ampicilina 2 gramos cada 4 horas, a lo que se añadieron tuberculostáticos tras el resultado del ADA. La tincion de gram fue negativa pero el cultivo de LCR y dos hemocultivos fueron positivos para Listeria monocytogenes, sustituyéndose el tratamiento por Ampicilina 2 gramos cada 4 horas y Gentamicina $1,7 \mathrm{mg} / \mathrm{kg}$ cada 8 horas durante 15 dias. No se administraron corticoides. A las 72 horas se resolvió la afasia y el meningismo. La PL de control mostró una mejoría de los parámetros bioquímicos y negativización del cultivo. No obstante, presentó hemorragia digestiva alta recidivante secundaria a una gastropatía congestiva a consecuencia de la cual se produjo el exitus a los 39 días del ingreso.

Las formas de presentación de los cuadros meníngeos incluyen desde una simple febrícula con alteraciones de la personalidad hasta una forma fulminante con coma precoz. En una búsqueda efectuada en medline sólo se han encontrado dos casos de afasia en el curso o como forma de presentación de una meningitis por L. monocytogenes $(4,5)$. En cambio, es más frecuente en otras meningoencefalitis (6). Por otro lado, en los pacientes VIH positivos parece existir una cierta protección a esta infección, por lo que los casos comunicados son relativamente escasos (2). Se ha sugerido que la causa de la presencia de afasia podría deberse a una vasculitis de las arterias leptomeníngeas del lóbulo frontal izquierdo (5). Asimismo, es de destacar la presencia de un ADA elevado en el líquido cefalorraquídeo. La presencia de un ADA elevado orienta hacia una meningitis tuberculosa $(7,8)$, aunque también se ha descrito en la neurobrucelosis y en la meningitis piógena, aunque en este caso el resto de parámetros clínicos y analíticos orientan hacia la afectación bacteriana (7). En cambio, en pacientes VIH, la utilidad del ADA se ha puesto en entredicho, puesto que se ha encontrado una baja sensibilidad y existen falsos positivos en casos de afectación neurológica por CMV y criptococo (9). En resumen, creemos que ante un cuadro de fiebre y focalidad neurológica en pacientes de riesgo, como en portadores del VIH o en hepatópatas crónicos, la meningitis por Listeria monocytogenes debería entrar en el diagnóstico diferencial (10). Asimismo, se debe tener en cuenta la posibilidad de falsos positivos en la determinación de ADA, lo que podría inducir a confusión con una meningitis tuberculosa.

\section{Milagros Montero, A. Supervía Caparrós, J. L. Gimeno- Bayón Cobos, A. Aguirre Tejedo, J. Gutiérrez Cebollada}

Servicio de Medicina Interna y Enfermedades Infecciosas y Servicio de Urgencias. Hospital del Mar. Barcelona 
1. Myolinakis E, Hohmann EL, Calderwood SB. Central nervous system infection with Listeria monocytogenes. 33 years' experience at a general hospital and review of 776 episodes from the leterature. Medicine 1998; 77: 313-336.

2. Alcoba Leza M, Carro Fernández JA, Pérez Simón MR, Guerra Laso J, Alonso Fernández A, Arto Millán L. Meningitis por Listeria monocytogenes en el adulto en España. Presentación de 10 casos y revisión de la literatura. Rev Clin Esp 2002; 202: 638-643.

3. Seidel Padilla V, Martínez-Roig A, Esteban Torne E, Herrero Pérez S, López Segura N, Santos Solis A, Bonet Alcaina M. Meningitis por Listeria monocytogenes en una niña inmunocompetente. An Pediatr (Barc) 2004; 60: 184-185.

4. Staudinger R, Levine D, Swaminathan B, Zagzag D. Neurolisteriosis presenting as recurrent transient ischemic attacks. Ann Neurol 2000; 48: 661-665.

5. Gerl A, Mittermuller J, Bise K, Wilmanns W. Listeriosis in malignant diseases. Dtsch Med Wochenschr 1991; 116: 1144-1148.

6. Hardin SG, Erwin PC, Patterson L, New D, Graber C, Halford SK. Clinical comparisons of La Crosse encephalitis and enteroviral central nervous system infections in a pediatric population: 2001 surveillance in East Tennessee. Am J Infect Control 2003; 31: 508-510.

7. López Cortés LF, Cruz Ruiz M, Gómez Mateos J, Jiménez Hernández D, Jiménez Mejias E, Pachón J, et al. Adenosine deaminase activity in the CSF of patients with aseptic meningitis: utility in the diagnosis of tuberculous meningitis or neurobrucellosis. Clin Infect Dis 1995; 20: 525-530.

8. Choi SH, Kim YS, Bae IG, Chung JW, Lee MS, Kang JM, et al. The possible role of cerebrospinal fluid adenosine deaminase activity in the diagnosis of tuberculous meningitis in adults. Clin Neurol Neurosurg 2002; 104: 10-15.

9. Corral I, Quereda C, Navas E, Martín Dávila P, Pérez Elias MJ, Casado JL, et al. Adenosine deaminase activity in cerebrospinal fluid of HIVinfected patients: limited value for diagnosis of tuberculous meningitis. Eur J Microbiol Infect Dis 2004; 23: 471-476.

10. Jurado RL, Farley MM, Pereira E, Harvey RC, Schuchant A, Wenger JD, Stephens DS. Increased risk of meningitis and bacteriemia due to Listeria Monocytogenes in patients with Human Inmunodeficiency virus infection.Clin Infect Dis 1993; 17: 224-227.

\section{Pileflebitis idiopática en paciente con mutación del factor V Leiden}

\section{Sr. Director:}

La pileflebitis o tromboflebitis séptica de la vena porta es una complicación poco frecuente, aunque muy grave, de las infecciones intraabdominales que puede preceder al desarrollo de abscesos hepáticos. La causa más frecuente es la diverticulitis, seguida de la apendicitis y la enfermedad inflamatoria intestinal (1-4). La vena mesentérica superior se afecta sólo en el 34\% de los casos y puede ocasionar isquemia intestinal, infarto o muerte (4). La forma idiopática es rara, representa un tercio de los casos, y se presenta como un cuadro de dolor abdominal inespecífico $(5,6)$. Presentamos un caso de pileflebitis idiopática con afectación de la vena mesentérica superior en una paciente portadora de una mutación del factor V Leiden.

Mujer de 28 años de edad con antecedentes de parto natural hace diez meses, fumadora de 20 cigarrillos/día y a tratamiento con anticonceptivos orales ( $3 \mathrm{mg}$ de drospirenona/ $30 \mu \mathrm{grs}$ de etinilestradriol) que consulta por un cuadro de tres semanas de evolución de dolor abdominal, náuseas, vómitos, escalofríos y fiebre termometrada de hasta $40^{\circ} \mathrm{C}$, que cede con antitérmicos.
En la exploración física presentaba: $\mathrm{T}^{\mathrm{a}} 38,7{ }^{\circ} \mathrm{C}$, PA125/80 mmHg, P109 lpm y un abdomen doloroso a la palpación en epigastrio y región periumbilical izquierda, sin signos de irritación peritoneal, siendo el resto de la exploración por aparatos anodina. En las pruebas complementarias destacaban 15050 leucocitos ( $85 \%$ neutrófilos), bilirrubina $0,9 \mathrm{mg} / \mathrm{dl}$, GOT $16 \mathrm{U} / \mathrm{L}$, GPT 21 U/L, GGT $290 \mathrm{U} / \mathrm{L}$, fosfatasa alcalina $210 \mathrm{U} / \mathrm{L}$, protrombina $66 \%$, fibrinógeno $754 \mathrm{mg} / \mathrm{dL}$ y VSG de $91 \mathrm{~mm}$ en la $1^{\mathrm{a}}$ hora. La analítica elemental de orina, la radiografía de tórax y abdomen eran normales y los hemocultivos negativos. La ecografía y el TAC abdominal demostraron la existencia de trombosis de la vena mesentérica superior hasta su confluencia con la vena esplénica, trombosis de la vena porta principal y de su rama izquierda. La enteroclisis y la colonoscopia resultaron normales. El estudio de la coagulación objetivó la presencia de mutación heterocigota del factor V Leiden (1691G/1691A) y resistencia a la proteína C activada. Se realizó tratamiento antibiótico con piperacilina-tazobactam, durante 4 semanas, y tratamiento anticoagulante, inicialmente con heparina sódica y posteriormente con acenocumarol.

La pileflebitis es una complicación poco frecuente de las infecciones intraabdominales con una mortalidad del 11 al $32 \%$ $(1,4,7)$. La causa más frecuente es la diverticulitis, seguida de la apendicitis y la enfermedad inflamatoria intestinal (1-4). La forma idiopática representa un tercio de los casos (1) y se asocia a alteraciones de los factores de la coagulación, enfermedades malignas o sida (4-7). El agente etiológico más frecuente es el Bacteroides spp., sobre todo B. fragilis, seguido por los bacilos gramnegativos y estreptococos aerobios. Los hemocultivos son positivos en el 23 al $88 \%$ de los casos $(1,4)$. Debe sospecharse ante un cuadro de dolor abdominal, fiebre y escalofríos que se acompañe de leucocitosis, aunque pueden ser normales o incluso presentar leucopenia, alteración de la coagulación y elevación de las enzimas hepáticas, especialmente de la fosfatasa alcalina de tres a cuatro veces su valor y de la GGT de cinco a diez veces (1$4,6,8)$. La ictericia es infrecuente a menos que se asocie a colangitis o abscesos hepáticos (1). El diagnóstico precisa la demostración de trombosis de la vena porta en un paciente febril. La ecografía abdominal suele mostrar la presencia de material ecogénico en el interior de la vena porta y puede utilizarse para evaluar la progresión o detectar la recanalización. El TAC se ha utilizado para confirmar el diagnostico de sospecha y es útil para descartar la presencia de focos infecciosos intraabdominales así como para detectar la presencia de abscesos hepáticos $(2,3,6,8)$.

El tratamiento antibiótico debe ser efectivo frente a bacilos gramnegativos y anaerobios, especialmente B. fragilis, y administrarse durante 4 a 6 semanas $(1,2,4,6)$. Se han descrito casos de colocación de un catéter en la vena porta para el drenaje de la tromboflebitis supurada con buenos resultados (5). En caso de existir abscesos hepáticos la asociación de antibióticos y drenaje percutáneo parece el tratamiento más indicado, con una tasa de supervivencia global del 90\% (9). Aunque aún no existe consenso sobre el tratamiento anticoagulante, la mayoría de los autores lo recomiendan en caso de que exista un estado de hipercoagulabilidad, afectación de la vena mesentérica superior, extensión de la trombosis o fiebre persistente que no responde al tratamiento antibiótico $(2,4,8,10)$. En el caso que presentamos se realizó tratamiento anticoagulante, inicialmente con heparina sódica y posteriormente con acenocumarol, presentando en el momento del alta recanalización de la rama izquierda de la vena porta y de la vena mesentérica superior.

R. López Rodríguez, C. Martínez Rey, J. Campos Franco, M. R. Alende Sixto, J. A. Torre Carballada

Servicio de Medicina Interna. Complejo Hospitalario Universitario de Santiago de Compostela. Santiago de Compostela. A Coruña 